\title{
DEVIATIONS OF TRAJECTORY AVERAGES AND THE DEFECT IN PESIN'S FORMULA FOR ANOSOV DIFFEOMORPHISMS
}

\author{
STEVEN OREY AND STEPHAN PELIKAN
}

\begin{abstract}
A large deviation theorem at the Donsker Varadhan level three is obtained for the convergence of trajectory averages of Anosov diffeomorphisms. It is possible to provide an explicit description of the rate function.
\end{abstract}

\section{INTRODUCTION}

This paper is concerned with the convergence of trajectory averages of certain smooth dynamical systems. If $f: M \mapsto M$ is a transitive Anosov diffeomorphism of a manifold $M$, the results of Bowen [B] imply the following.

For each continuous function $\varphi: M \mapsto \mathbf{R}$ the trajectory averages of $\varphi$, that is the sequence $\frac{1}{n} \sum_{0}^{n-1} \varphi\left(f^{j}(x)\right)$, converge to a constant for Lebesgue almost every initial point $x \in M$. This constant is $\int \varphi d \mu$, where $\mu$ is the Bowen-RuelleSinai measure. An equivalent assertion is that the measures $\frac{1}{n} \sum_{0}^{n-1} \delta_{f^{j}(x)}$ converge weakly to $\mu$ for Lebesgue almost all $x$.

We are concerned with estimating the probability that the $n$th trajectory average differs from $\int \varphi d \mu$; and in particular, with estimating the rate of exponential decay of the numbers

$$
m\left\{x: \frac{1}{n} \sum_{0}^{n-1} \varphi\left(f^{j}(x)\right) \in A\right\} \quad \text { as } n \mapsto \infty .
$$

Here $m$ is a normalized Lebesgue measure and $A \subset \mathbf{R}$ is some Borel set.

Our first conclusion, Corollary 4.7 , is that for each continuous function $\varphi$ there is a lower semicontinuous $K^{\varphi}: \mathbf{R} \mapsto[0, \infty]$ which describes these decay rates in the sense that

$$
\limsup _{n \rightarrow \infty} n^{-1} \log m\left\{x: \frac{1}{n} \sum_{0}^{n-1} \varphi\left(f^{j}(x)\right) \in A\right\} \leq-\inf \left\{K^{\varphi}(t): t \in A\right\}
$$

Received by the editors October 6, 1986 and, in revised form, April 1, 1988.

1980 Mathematics Subject Classification (1985 Revision). Primary 60F10, 58F15.

Key words and phrases. Anosov diffeomorphism, trajectory average, large deviations.

Supported in part by National Science Foundation Grant MCS83-01080.

Supported in part by National Science Foundation Grant DMS 8601014. 
if $A \subset \mathbf{R}$ is closed and

$$
\liminf _{n \mapsto \infty} n^{-1} \log m\left\{x: \frac{1}{n} \sum_{0}^{n-1} \varphi\left(f^{j}(x)\right) \in A\right\} \geq-\inf \left\{K^{\varphi}(t): t \in A\right\}
$$

if $A \subset \mathbf{R}$ is open.

The second conclusion presented is that there is a lower semicontinuous function $K$ defined on the set $\mathscr{M}(M)$ of probability measures on $M$, and taking values in $[0, \infty]$ so that

$$
K^{\varphi}(t)=\inf \left\{K(\nu): \nu \in \mathscr{M}(M) \text { and } \int \varphi d \nu=t\right\} .
$$

In addition, the function $K$ has an explicit representation

$$
K(\nu)= \begin{cases}-h_{\nu}(f)-\int \varphi^{u} d \nu & \text { if } \nu \text { is invariant under } f \\ +\infty & \text { otherwise. }\end{cases}
$$

Here $h_{\nu}(f)$ is the metric entropy of $f$ and $\varphi^{u}(x)=-\log \left|\operatorname{det} D f_{\mid E^{u}}\right|$ is the negative logarithm of the determinant of $D f$ restricted to the unstable subspace $E^{u} \subset T M$ determined by $f$.

Recognizing $-\int \varphi^{u} d \nu$ as the sum of the positive $\nu$-Lyapunov exponents $\chi_{i}^{\nu}$ of $f$, (ii) can be rewritten as

$$
h_{\nu}(f)+K(\nu)=\sum\left[\chi_{i}^{\nu}\right]^{+} .
$$

In this formulation the "rate function" $K$ can be seen to measure the defect in "Pesin's formula" ([P]), apparently due originally to G. A. Margolis, which says,

$$
h_{\nu}(f) \leq \sum\left[\chi_{i}^{\nu}\right]^{+}
$$

In the language of the theory of large deviations as set forth in [V], what we show is that the sequence $\Phi_{n}(\cdot)=m\left\{x: \frac{1}{n} \sum_{0}^{n-1} \delta_{f^{j}(x)} \in \cdot\right\}, n=1,2, \ldots$, of measures on $\mathscr{M}(M)$ satisfies a large deviation principle with rate function given by (ii). This result was suggested to us by remarks in [T].

Some concepts of large deviation theory are summarized in $\S 2$. In $\S 3$, the facts required concerning hyperbolic dynamical systems are recalled, and $\S 4$ is devoted to the proofs of our main results.

\section{Preliminaries on RATE FUNCTIONS AND ShiFtS}

Rate functions. A generalized dynamical system is $(X, T, \mu)$ where $X$ is a topological space, $T: X \mapsto X$ is a homeomorphism, and $\mu$ is a Borel probability measure. Note that we do not require that $\mu$ be $T$-invariant. The probability measures on $X$ will be denoted $\mathscr{M}(X)$ and those invariant under $T$ by $\mathscr{M}_{T}(X)$.

If $\varphi: X \mapsto V$ is a map of $X$ into a convex subset of a topological linear space, set $S_{n}(\varphi)(x)=\frac{1}{n} \sum_{j=0}^{n-1} \varphi \circ T^{j}(x)$. For a subset $A \subset V$ define

$$
\lambda_{*}(A)=\liminf _{n \mapsto \infty} n^{-1} \log \mu\left(\left[x: S_{n}(\varphi)(x) \in A\right]\right)
$$


and

$$
\lambda^{*}(A)=\limsup _{n \mapsto \infty} n^{-1} \log \mu\left(\left[x: S_{n}(\varphi)(x) \in A\right]\right) .
$$

A function $K: V \mapsto[0, \infty]$ is a rate function for $(X, T, \mu)$ and $\varphi$ if the following conditions hold:

(1) $-\inf \{K(a): a \in A\} \leq \lambda_{*}(A)$ for each open set $A \subset V$,

(2) $-\inf \{K(a): a \in A\} \geq \lambda^{*}(A)$ for each closed set $A \subset V$,

(3) $K$ is lower semicontinuous,

(4) $\{a: K(a) \leq t\}$ is compact for each $t$.

If $K$ is a rate function for $\left(X, T, \mu^{\gamma}\right)$ and $\varphi$ for every $\gamma$ in some index set $\Gamma$, it is a uniform rate function for the family if the limits in the inequalities (1) and (2) above hold uniformly for $\gamma \in \Gamma$.

When a rate function exists, it is unique. In our work below, $V$ will be compact and condition (4) will be trivially true.

We are particularly interested in establishing the existence of rate functions where $\varphi: X \mapsto \mathbf{R}$ is a continuous "observation". One way to establish the existence of rate functions for all $\varphi \in C(X, \mathbf{R})$ simultaneously is to show that there is a rate function for the system $(X, T, \mu)$ and the function $\delta: X \rightarrow$ $M(X)$ defined by $\delta(x)=\delta_{x}=$ point mass at $x$, and then apply Proposition 2.1 below. This proposition is a variant of the "contraction principle" of [V], see [O, Proposition 1.2].

2.1. Proposition. For $i=1,2$ let $\left(X_{i}, T_{i}, \mu_{i}^{x_{i}}\right), x_{i} \in X_{i}^{\prime}$, be a family of $d y$ namical systems, where $X_{i}^{\prime} \subset X_{i}$. Suppose $\phi_{i}: X_{i} \mapsto V_{i}$ are maps where $V_{i}$ is a convex subset of a metrizable linear space. Suppose $X_{1}$ is compact and that there exists a measurable map $L: X_{1} \mapsto X_{2}$ so that $\mu^{x_{i}} \circ L^{-1}=\mu^{L x_{i}}$ for all $x_{i} \in X_{i}^{\prime}$ and $L\left(X_{1}^{\prime}\right)=X_{2}^{\prime}, L \circ T_{1}=T_{2} \circ L$. Also suppose there is a bounded linear map $M: V_{1} \mapsto V_{2}$ so that $\phi_{2} \circ L=M \circ \phi_{1}$. Then, if $K_{1}$ is a uniform rate function for $\left(X_{1}, T_{1}, \mu_{1}^{x_{1}}\right)$, and $\delta_{1}$ (for $\left.x_{1} \in X_{1}^{\prime}\right)$, the function $K_{2}$ defined by

$$
K_{2}(y)=\left\{\begin{array}{l}
\inf \left\{K_{1}(x): M x=y\right\} \\
+\infty \text { if } y \notin \operatorname{range}(M)
\end{array}\right.
$$

is a uniform rate function for $\left(X_{2}, T_{2}, \mu_{2}^{x_{2}}\right)$, and $\delta_{2}\left(\right.$ for $\left.x_{2} \in X_{2}^{\prime}\right)$.

In case $(X, T, \mu)$ and the function $x \mapsto \delta_{x}$ has a rate function, we will simply say that the system has a rate function. In this case the rate function can be shown to have the value $+\infty$ off of the set $\mathscr{M}_{T}(X)$.

2.2. Corollary. Suppose $N>0$ and that the system $\left(X, T^{N}, \mu\right)$ has a rate function $K_{1}$. Then the system $\left(X, T^{N}, \mu\right)$ and the function

$$
x \mapsto N^{-1}\left(\delta_{x}+\delta_{T x}+\cdots+\delta_{T^{N-1} x}\right)
$$

has a rate function $K_{2}$, and $K_{2}(\nu)=K_{1}(\nu)$ if $\nu \in \mathscr{M}_{T^{N}}(X)$. 
Proof. Apply Proposition 2.1 to the following diagram:

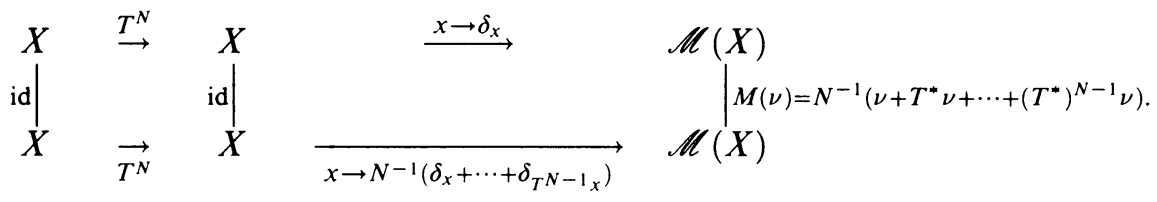

Note that $M(\nu)=\nu$ on $\mathscr{M}_{T^{N}}(X)$.

For future reference we record the following, which is a consequence of the lower semicontinuity of rate functions and the fact that the average of the function $\phi(x)=N^{-1}\left(\delta_{x}+\cdots+\delta_{T^{N-1} x}\right)$ along trajectories of $T^{N}$ is also an average of the function $x \rightarrow \delta_{x}$ along trajectories of $T$. That is, for each set $A$, $\left\{x: n^{-1} \sum_{0}^{n-1} \phi\left(T^{N j}(x)\right) \in A\right\}=\left\{x:(N n)^{-1} \sum_{0}^{N n-1} \delta_{T^{j}(x)} \in A\right\}$.

2.3. Proposition. If $\left(X, T^{N}, \mu\right)$ has a rate function $K_{1}$ for

$$
\phi(x)=N^{-1}\left(\delta_{x}+\cdots+\delta_{T^{N-1} x}\right),
$$

then $(X, T, \mu)$ has a rate function $K_{2}$ for the function $\delta_{x}$, and $K_{2}=N^{-1} K_{1}$.

Shifts and measures. When $M=\{1,2, \ldots, n\}$ is a finite set given the discrete topology, form $\Omega=\prod_{-\infty}^{\infty} M$, and give $\Omega$ the product topology and product Borel algebra $\mathscr{F}$. Denote by $\mathscr{F}_{m, n}$ for $m<n$ and $m, n \in \mathbf{Z} \cup\{-\infty, \infty\}$ the smallest sub- $\sigma$-algebra of $\mathscr{F}$ with respect to which the coordinate functions $\omega \mapsto \omega_{i}$ for $m \leq i \leq n$ are measurable. The shift homeomorphism on $\Omega$ is $\sigma: \Omega \mapsto \Omega$ defined by $[\sigma(\omega)]_{n}=\omega_{n+1}$.

If $A$ is an $n \times n$ matrix with each entry equal to 0 or 1 , define a closed $\sigma$-invariant subset of $\Omega$ by $\Sigma_{A}=\left\{\omega \in \Omega: A_{\omega_{i} \omega_{i+1}}=1\right.$ for all $\left.i \in \mathbf{Z}\right\}$. Such subsets, equipped with the relative $\sigma$-algebras $\mathscr{F}_{m, n}$ are called subshifts of finite type.

For $\mu \in \mathscr{M}(\Omega)$, denote by $\mu\left(\cdot \mid \mathscr{F}_{m, n}\right)(\omega)$ the conditional probabilities of $\mu$ given the $\sigma$-algebra $\mathscr{F}_{m, n}$. A version $\mu^{*}(\cdot)(\omega)$ of $\mu\left(\cdot \mid \mathscr{F}_{m, n}\right)(\omega)$ is called a family of regular conditional probabilities given $\mathscr{F}_{m, n}$ if for each $\omega \in \Omega$ the function $A \mapsto \mu^{*}(A)(\omega)$ is a probability measure on $\mathscr{F}$. We will always use the notation $\nu(\cdot \mid G)(x)$ for conditional probabilities and $\nu^{*}(\cdot)(x)$ or $\nu_{x}^{*}(\cdot)$ for regular condtional probabilties-the $\sigma$-algebra being understood.

Gibbs measures. Equip $\Omega$ (or $\Sigma_{A}$ ) with a metric by defining $d(\omega, \eta)=$ $\sum_{-\infty}^{\infty} 2^{-|i|} \delta_{\omega_{i}}^{\eta_{i}}$. If $\phi: \Omega \mapsto \mathbf{R}$ is Hölder continuous in this metric, there is a Gibbs measure $\mu_{\phi}$ associated with $\phi$. It is the unique $\sigma$-invariant measure which satisfies the property:

There exist positive constants $C_{1}$ and $C_{2}$ and a number $P(\phi)$ so that for each $\omega \in \Omega$

$$
C_{1} \leq \frac{\mu_{\phi}\left(C^{n}(\omega)\right)}{\exp \left(-P(\phi) n+\sum_{0}^{n-1} \phi\left(\sigma^{j}(\omega)\right)\right)} \leq C_{2}
$$


where $C^{n}(\omega)=\left\{\eta \in \Omega: \eta_{i}=\omega_{i}\right.$ for $\left.i=0,1,2, \ldots, n-1\right\}$. For a reference on Gibbs measures, see [B].

We will require the following result:

2.2. Theorem [OP, Theorem 3.2]. Let $\left(\Sigma_{A}, \sigma, \mu\right)$ be a subshift of finite type with $\mu$ a Gibbs measure for some Hölder continuous function $\phi: \Sigma_{A} \mapsto \mathbf{R}$. There exists a family $\mu^{*}(\cdot)(\omega), \omega \in \Sigma_{A}$, of regular conditional probabilities of $\mu$ given $\mathscr{F}_{-\infty, 0}$ such that the function $K: \mathscr{M}\left(\Sigma_{A}\right) \mapsto[0, \infty]$ given by

$$
K(\nu)=\left\{\begin{array}{l}
-h_{\nu}(\sigma)-\int \phi d \nu+P(\phi), \quad \nu \in \mathscr{M}_{\sigma}\left(\Sigma_{A}\right), \\
+\infty \text { otherwise }
\end{array}\right.
$$

is a uniform rate function for the family of systems $\left(\Sigma_{A}, \sigma, \mu_{\omega}^{*}(\cdot)\right), \omega \in \Sigma_{A}$.

It is the purpose of the next two sections to use the methods of symbolic dynamics to "transfer" this result to the setting of Anosov diffeomorphisms.

\section{ANOSOV DIFFEOMORPHISMS}

Let $M$ be a compact Riemannian manifold and $f: M \mapsto M$ a transitive $C^{2}$ Anosov diffeomorphism. That is, suppose the tangent bundle to $M$ splits in a continuous sum $T M=E^{s}+E^{u}$ where there exist constants $C>0$ and $\lambda \in(0,1)$ so that

(i) $\left\|D f^{n}(x) \cdot v\right\| \leq C \lambda^{n}\|v\|$ for all $n \geq 0$ and $v \in E_{x}^{s}$,

(ii) $\left\|D f^{n}(x) \cdot v\right\| \leq C \lambda^{n}\|v\|$ for all $n \leq 0$ and $v \in E^{u}$.

To fix notation we record some facts concerning Anosov diffeomorphisms.

(1) Stable manifolds. Through each $x \in M$ pass two $C^{1}$ submanifolds $W_{x}^{s}$ and $W_{x}^{u}$ tangent at $x$ to the subspaces $E_{x}^{s}$ and $E_{x}^{u}$. These submanifolds are the collections of points whose forward (backward) orbit under $f$ approaches the forward (backward) orbit of $x$. The foliations of $M$ given by these manifolds are invariant under $f$ (leaves are mapped to leaves), and transverse.

(2) Local product structure. The local stable and unstable manifolds of $x$ are

$$
W_{\varepsilon}^{s}(x)=\left\{y \in W_{x}^{s}: d\left(f^{j} x, f^{j} y\right) \leq \varepsilon \text { for all } j=0,1,2, \ldots\right\}
$$

and

$$
W^{u}(x)=\left\{y \in W^{u}: d\left(f^{j} x, f^{j} y\right) \leq \varepsilon \text { for all } j=0,-1,-2, \ldots\right\} .
$$

There exist $\varepsilon$ and $\delta$ so that if $x, y \in M$ satisfy $d(x, y)<\delta$, then $W_{\varepsilon}^{u}(x) \cap$ $W_{\varepsilon}^{s}(x)$ consists of exactly one point, denoted $[x, y]$. Thus one obtains a homeomorphism from a neighborhood of $x$ in $W^{u}(x)$ to a neighborhood of $y$ in $W^{u}(y)$ for $y$ in $W^{s}(x)$ near enough $x$, by setting

$$
h_{x, y}(p)=W_{\varepsilon}^{s}(p) \cap W_{\varepsilon}^{u}(y)=[p, y] .
$$

A theorem of Anosov [A] states that this homeomorphism is absolutely continuous with respect to Lebesgue measure (induced on $W_{\varepsilon}^{u}(x)$ and $W_{\varepsilon}^{u}(y)$ by restricting the metric of $M$ to the tangent spaces of these manifolds and extending the resulting volume form to a measure). If $m_{x}$ and $m_{y}$ are the Lebesgue 
measures on $W^{u}(x)$ and $W^{u}(y)$, define a new measure $\bar{m}$ on a neighborhood $N$ of $x$ in $W^{u}(x)$ by $\bar{m}(A)=m_{y}\left(h_{x, y}(A)\right)$. Anosov's theorem says that $\bar{m}$ and $m_{x}$ are equivalent, and that in fact, the densities of one with respect to the other are bounded and depend continuously on the point $y$.

(3) Markov partitions. A set $R \subset M$ is called a rectangle if $[x, y] \in R$ whenever $x$ and $y$ are in $R$ and if, in addition, $R=\operatorname{closure(interior}(R))$. If $R$ is a rectangle, the boundary of $R$ comes in two pieces

$$
\begin{aligned}
& \partial^{e} R=\left\{x \in R: x \notin \operatorname{int}\left(W_{\varepsilon}^{s}(x) \cap R\right)\right\}, \\
& \partial^{c} R=\left\{x \in R: x \notin \operatorname{int}\left(W_{\varepsilon}^{u}(x) \cap R\right)\right\} .
\end{aligned}
$$

A Markov partition of $M$ is a cover of $M$ by rectangle $R_{1}, R_{2}, \ldots, R_{n}$ so that

(i) $\operatorname{int}\left(R_{i}\right) \cap \operatorname{int}\left(R_{j}\right)$ is empty if $i \neq j$,

(ii) $f\left(\partial^{c} R_{i}\right) \subset \bigcup_{j} \partial^{c} R_{j}$, for all $i$,

(iii) $f^{-1}\left(\partial^{e} R_{i}\right) \subset \bigcup_{j} \partial^{e} R_{j}$, for all $i$.

For a detailed account of the existence of stable manifolds, local coordinates and Markov partitions, consult [B] and the references found there. The theory of Markov partitions provides, for each sufficiently fine Markov partition $\mathscr{R}=\left\{R_{1}, \ldots, R_{n}\right\}$ of $M$, a subshift of finite type $\Sigma_{A}$, and a continuous map $\pi: \sigma_{A} \mapsto M$ (onto) so that $\pi \circ \sigma=f \circ \pi$. The subshift and map are, roughly, defined as follows. For $A$ take the $n \times n$ matrix defined by $a_{i j}=0$ or 1 according to whether $\operatorname{int}\left(R_{j}\right) \cap \operatorname{int}\left(f\left(R_{i}\right)\right)$ is empty or not. For $\omega \in \Sigma_{A}$, the set $\bigcap_{\mathrm{Z}} f^{-j}\left(R_{\omega_{j}}\right)$ contains exactly one point $x$, and one defines $\pi(\omega)=x$. One can show $\pi$ is well defined, continuous, and onto. In addition, $\pi$ is $1-1$ except at those points $\omega$ which map to the first category set $\bigcup_{\mathrm{z}} f^{j}\left(\bigcup_{i} \partial R_{i}\right)$.

Denote by $\xi$ the "partition" of $M$ into sets having the form $R_{i} \cap W_{\varepsilon}^{u}(x)$, for $R_{i} \in \mathscr{R}=\left\{R_{1}, \ldots, R_{n}\right\}$ a member of a generating Markov partition. Then $\xi$ is a partition of $M$ modulo a set having zero Lebesgue measure: different sets in $\xi$ can only intersect at a subset of $\bigcup_{i} \partial R_{i}$, which has Lebesgue measure zero. This fact was established by Sinai [S1] and by Ratner [Ra]. We can convert $\xi$ into a true partition by selecting a scheme for assigning points in the common boundaries of two sets $R_{i}$ and $R_{j}$ to one of these sets, and removing them from the other. In what follows, we will consider conditional probabilities given $\xi$. The theory of conditional probabilities given a partition (see [ME]) ccrresponds very closely to the theory of conditional probabilities given a $\sigma$ algebra. It will be convenient to continue to use the notation $\nu_{x}^{\xi}$ for a version of regular conditional probabilities given $\xi$ even when $\xi$ is a partition. This should cause no confusion. Under the map $\pi: \Sigma_{A} \mapsto M$, the algebra generated by $\xi$ correspond to $\mathscr{F}_{-\infty, 0}$.

(4) Conditional Lebesgue measure. If $m$ is (normalized) Lebesgue measure on $M$, the family of conditional measures of $m$ given $\xi$, denoted $m(\cdot \mid \xi)(x)$, is defined $m$-a.e. There is a second means by which we might try to construct 
such a family of conditional measures explicitly: restrict the metric of $M$ to $T W^{u}(x)$, and determine a volume form on $W^{u}(x)$, extend this volume to a measure, and finally restrict this measure to $C_{\xi}(x)=$ the set of $\xi$ containing $x$, and normalize it. Denote the result of this construction by $m_{x}^{*}$. To see that, for $m$-a.e. $x$, the measures $m_{x}^{*}$ and $m(\cdot \mid \xi)(x)$ restricted to $C_{\xi}(x)$ are equivalent, it suffices to work locally (that is, in one particular rectangle $R_{i}$ ), and to verify that there is a constant $K>1$ so that

$$
\int_{R} m_{x}^{*}\left(C_{\xi}(x) \cap A\right) d m(x) \in \int_{R} m\left(C_{\xi}(x) \cap A \mid \xi\right)(x) d m(x) \cdot\left[K^{-1}, K\right],
$$

independent of $x$, where $A \subset R_{i}$ is an arbitrary rectangle in $R$.

This would be immediate if the partition $\xi$ were a partition of a neighborhood of 0 in $\mathbf{R}^{n}$ into a family of $k$-dimensional "horizontal" planes. The general case can be reduced to this one by constructing a "rectifying" homeomorphism as follows. Pick $p \in R$ and let $\phi_{1}: \Delta^{1} \mapsto W^{s}(p), \phi_{2}: \Delta^{2} \mapsto W^{u}(p)$ be embeddings of disks $\Delta^{1} \subset \mathbf{R}^{k}, \Delta^{2} \subset \mathbf{R}^{n-k}$, where $k=\operatorname{dim}\left(W^{s}(p)\right)$, $n=\operatorname{dim}(M)$, so that $\operatorname{Range}\left(\phi_{1}\right)=W^{s}(p) \cap R_{i}$ and $\operatorname{Range}\left(\phi_{2}\right)=W^{u}(p) \cap R_{i}$. Extend these functions to $\Delta^{1} \times \Delta^{2}$ by setting $\phi(x, y)=\left[\phi_{2}(y), \phi_{1}(x)\right]$. Then Anosov's theorem says that for each fixed $x$ (or $y$ ) the map $y \mapsto \phi(x, y)$ (or $x \mapsto \phi(x, y)$ ) is absolutely continuous. This observation and the uniform hyperbolicity of $f$ suffice to complete the proof.

\section{The MAIN RESUlTS}

Let $f: M \mapsto M$ be a transitive $C^{2}$ Anosov diffeomorphism, $\mathscr{R}=\left\{R_{1}, \ldots\right.$, $R_{n}$ \} a generating Markov partition, and $\pi: \Sigma_{A(\mathscr{R})} \mapsto M$ be as in $\S 3$. We require that each set $R_{i}$ of the partition be so small that it lies entirely in one local coordinate neighborhood. Define $\phi^{u}(x)=-\log \left|\operatorname{det} D f(x)_{\mid E^{u}(x)}\right|$ and set $\bar{\varphi}=$ $\varphi^{u} \circ \pi$.

The function $\varphi^{u}$ is Hölder continuous [B, Lemma 4.6] and $\bar{\phi}$ inherits this property, since there exist constants $C$ and $\gamma$ so that for every $\omega, \eta \in \Sigma_{A}$,

$$
d(\pi(\omega), \pi(\eta)) \leq C(d(\omega, \eta))^{\gamma} .
$$

Thus $\bar{\varphi}$ determines a Gibbs measure $\bar{\mu}$. This measure is characterized as the unique $\sigma$-invariant measure on $\Sigma_{A}$ at which the supremum (over $\sigma$-invariant measures $\nu$ ) of the function $\nu \mapsto h_{\nu}(\sigma)+\int \bar{\phi} d \nu$ is attained.

Similarly, $\varphi^{u}$ determines a Gibbs measure $\mu$ characterized by

$$
\begin{aligned}
P\left(\varphi^{u}\right) & =\sup \left\{h_{\eta}(f)+\int \varphi^{u} d \eta: \eta \in \mathscr{M}_{f}(M)\right\} \\
& =h_{\mu}(f)+\int \varphi^{u} d \mu .
\end{aligned}
$$

One may refer to [B, $\S 10$ and Theorem 4.1]. The measure $\mu$ (denoted by $\mu^{+}$in [B]) is the Bowen-Ruelle-Sinai measure. In fact, $\mu=\pi^{*} \bar{\mu}, \bar{\mu}(\bar{\varphi})=\mu\left(\varphi^{u}\right)$, and 
$h_{\bar{\mu}}(\sigma)=h_{\mu}(f)$, so that $P(\bar{\varphi})=P\left(\varphi^{u}\right)$. For a proof see $[\mathrm{R}, \S 7.8]$. Furthermore, $P\left(\varphi^{u}\right)=0$. (See [B, Proposition 4.8].)

In this section we prove the following:

4.1. Theorem. Let $m$ be normalized Lebesgue measure on $M$. The generalized dynamical system $(M, f, m)$ has a rate function $K: \mathscr{M}(M) \mapsto[0, \infty]$ given by

$$
K(\nu)= \begin{cases}-h_{\nu}(f)-\int \varphi^{u} d \nu & \text { if } \nu \in \mathscr{M}_{f}(M), \\ +\infty & \text { otherwise. }\end{cases}
$$

The proof proceeds in a number of steps. We begin with an overview. By Theorem 2.2, there is a rate function which works uniformly for the measures $\mu^{*}(\cdot)(\omega)$, where $\mu^{*}$ is a family of regular conditional probabilities of $\bar{\mu}$ given $\mathscr{F}_{-\infty, 0}$.

We can construct a version of $\mu(\cdot \mid \xi)(x)$ by defining $\mu(A \mid \xi)(x)$ to be $\mu^{*}\left(\pi^{-1}(A)\right)\left(\pi^{-1}(x)\right)$ for $\mu$ almost every $x \in M$. This provides a rate function for $\mu(\cdot \mid \xi)(x)$ as in Lemma 4.2. A result known to Anosov [A] (see also [L]) says that $\mu(\cdot \mid \xi)(x)$ and $m_{x}^{*}$ are equivalent. Using the absolute continuity of the maps $h_{x y}$ with respect to $m_{x}^{*}$ and $m_{y}^{*}$, we obtain estimates on $m_{x}^{*}\left\{y: \frac{1}{n} \sum_{0}^{n-1} \delta_{f^{j}(x)} \in A\right\}$ for $m$-almost every $x$ in $M$. Finally, the equivalence of $m_{x}^{*}$ and $m(\cdot \mid \xi)(x)$ is used to compute $m\left\{y: \frac{1}{n} \sum_{0}^{n-1} \delta_{f^{j}(x)} \in A\right\}$, which completes the proof.

To simplify notation, write $L_{n}(x)$ for $\frac{1}{n} \sum_{0}^{n-1} \delta_{f^{j}(x)}$. Let $D \subset M$ be the set of points $x \in M$ for which $\pi^{-1}(x)$ is not a singleton. That is, $D=\bigcup_{\mathbf{Z}} f^{j}(\partial \mathbf{R})$, where $\partial \mathscr{R}=\bigcup_{i} \partial R_{i}$. Recall that $\mu(D)=\bar{\mu}\left(\pi^{-1}(D)\right)=0$, as shown in [B, Theorem 4.1], and that $m(D)=0$, since $m(\partial \mathscr{R})=0$, as remarked above. Fix regular conditional probabilities $\mu^{*}(\cdot)(\omega)$ for $\bar{\mu}$ given $\mathscr{F}_{-\infty, 0}$, and some version of $\mu(\cdot \mid \xi)(x)$. For $B \subset M$, set

$$
\hat{\mu}_{x}^{\xi}(B)= \begin{cases}\mu(B \mid \xi)(x) & \text { if } x \in D, \\ \mu^{*}\left(\pi^{-1} B\right)\left(\pi^{-1} x\right) & \text { if } x \notin D .\end{cases}
$$

Then $\hat{\mu}_{x}^{\xi}$ is a version of $\mu(\cdot \mid \xi)(x)$.

4.2. Lemma. Let $\left(\mu_{x}^{\xi}\right)$ be a family of regular conditional probabilties of $\mu$ given $\xi$. There exists a set $V \subset M$ with $\mu(V)=1$ such that there is a uniform rate function $K$ for the systems $\left(M, f, \mu_{x}^{\xi}\right), x \in V$. If $\nu \in \mathscr{M}(M)$ satisfies $\nu(D)=0$, then

$$
K(\nu)= \begin{cases}-h_{\nu}(f)-\int \varphi^{u} d \nu & \text { if } \nu \in \mathscr{M}_{f}(M), \\ +\infty & \text { otherwise. }\end{cases}
$$

Proof. Since the Borel $\sigma$-field on $M$ is generated by a countable set and for any Borel set $A, \hat{\mu}_{x}^{\xi}(A)=\mu_{x}^{\xi}(A)$ for $\mu$-a.e. $x$, the measures $\hat{\mu}_{x}^{\xi}$ and $\mu_{x}^{\xi}$ coincide for all $x$ outside a $\mu$-null set $F$. It will suffice to prove the lemma 
for the family $\hat{\mu}_{x}^{\xi}$ with $V=M-D$. The result for $\mu_{x}^{\xi}$ then follows with $V=M-(D \cup F)$.

First apply Theorem 2.2 to the family $\left(\Sigma_{A}, \sigma, \mu_{\omega}^{*}(\cdot)\right)$ with $\mu_{\omega}^{*}$ as in Theorem 2.2. We have already noted that $P(\bar{\phi})=0$, and so we obtain the uniform rate function

$$
K_{1}(\nu)= \begin{cases}-h_{\nu}(\sigma)-\int \bar{\varphi} d \bar{\nu} & \text { if } \nu \in \mathscr{M}_{\sigma}\left(\Sigma_{A}\right), \\ +\infty & \text { otherwise. }\end{cases}
$$

Now apply Proposition 2.1 to the families $\left(\Sigma_{A}, \sigma, \mu_{\omega}^{*}(\cdot)\right), \omega \in \pi^{-1}(M-D)$ and $\left(M, f, \hat{\mu}_{x}^{\xi}\right), x \in M-D$, where $V_{1}=\mathscr{M}\left(\Sigma_{A}\right), V_{2}=\mathscr{M}(M), \phi_{1}$ is the map $\delta: \Sigma_{A} \mapsto \mathscr{M}\left(\Sigma_{A}\right)$, (that is $\delta(\omega)=\delta_{\omega}$ ), and $\phi_{2}$ is the map $x \mapsto \delta_{x}$. The function $L: \Sigma_{A} \rightarrow M$ is $\pi$. The function $M: V_{1} \mapsto V_{2}$ is $\pi^{*}$, defined by $\pi^{*}(\bar{\nu})=\nu \circ \pi^{-1}$. Since $\pi$ is continuous, so is $\tau^{*}$. Since $K_{1}$ is uniform on $\Sigma_{A}$ for $\omega \in \Sigma_{A}-\pi^{-1}(D)$, and $\pi^{*}\left(\hat{\mu}_{\omega}^{*}\right)=\mu_{\pi(\omega)}^{\xi}$, we obtain a uniform rate function $K$ for $\left(M, f, \hat{\mu}_{x}^{\xi}\right), x \in M-D$. For $\nu \in \mathscr{M}_{f}(M), \bar{\nu} \in \mathscr{M}_{\sigma}(M)$ with $\pi^{*} \bar{\nu}=\nu$, one has $\int \bar{\varphi} d \bar{\nu}=\int \varphi d \nu$. Suppose that $\nu(D)=0$. Then $\pi$ provides a measure theoretical isomorphism between the systems $(M-D, f, \nu)$ and $\left(\Sigma_{A}-\pi^{-1}(D), \sigma, \bar{\nu}\right)$. Consequently, $h_{\nu}(f)=h_{\bar{\nu}}(\sigma)$, and the expression for $K$ given in the statement of the lemma follows from the expression for $K_{1}$.

4.3. Lemma. Relation (1) holds for any $\nu \in \mathscr{M}_{f}$.

Proof. If $\nu \in \mathscr{M}_{f}$ but $\nu(D)=0$ is not assumed, the argument used in Lemma 4.2 gives

$$
K(\nu)=-\bar{h}_{\nu}(f)-\int \varphi^{u} d \nu
$$

where $\bar{h}_{\nu}(f):=\sup \left\{h_{\bar{\nu}}(\sigma): \pi^{*}(\bar{\nu})=\nu\right\}$. The definition of $\bar{h}_{\nu}(f)$ involves the mapping $\pi$ and hence the Markov partition $\mathscr{R}$; however, formula (3) shows that in fact it is independent of the choice of $\mathscr{R}$. To prove the lemma we must show that $h_{\nu}(f)=\bar{h}_{\nu}(f)$. If $\nu \in \mathscr{M}_{f}$ and $\bar{\nu} \in \mathscr{M}_{\sigma}$ with $\pi^{*}(\bar{\nu})=\varphi$, one sees easily that $h_{\bar{\nu}}(\sigma) \geq h_{\nu}(f)$, so that only the inequality

$$
h_{\nu}(f) \geq \bar{h}_{\nu}(f)
$$

must be verified.

Using the fact that $h_{\nu}(f)$ and $h_{\bar{\nu}}(\sigma)$ are affine functions of $\nu$ and $\bar{\nu}$ respectively, we see that the set of $\nu$ for which (1) holds is convex. We exploit this observation repeatedly. In addition, either because of Proposition 2.3 or because of the property of entropy that says if $n>0$ then $h_{\nu}\left(f^{n}\right)=n h_{\nu}(f)$, we may verify the lemma with $f$ replaced by some iterate $f^{m}$.

Any $\nu \in \mathscr{M}_{f}$ can be written as a convex combination of $\nu_{1}$ and $\nu_{2}$ in $\mathscr{M}_{f}$ with $\nu_{1}(D)=0$ and $\nu_{2}(D)=1$. In fact, we may assume that $\nu_{2}(\partial \mathscr{R})=1$. To see this, let $S_{0}=\partial^{s}(\mathscr{R})$ and $U_{0}=\partial(\mathscr{R}) \backslash S_{0}$. Set $S_{j}=f^{j}\left(S_{0}\right)$, so that $S_{j+1} \subset S_{j}$. The invariance of $\nu_{2}$ implies that $\nu_{2}\left(S_{j+1} \backslash S_{j}\right)=0$. It follows that 
$\bigcup_{j \in \mathbf{Z}} S_{j}$ differs from $S_{\infty}=\bigcap_{j \in \mathbf{Z}} S_{j}$ by a $\nu_{2}$-null set. After a similar argument concerning $U_{0}$ we see that $D=S_{-\infty} \cup U_{\infty}$ differs from $S_{\infty} \cup U_{-\infty} \subset \partial \mathscr{R}$ by a $\nu_{2}$-null set. Since $\nu_{1}$ satisfies (1) by Lemma 4.2, we need only show that (4), or equivalently that $(1)$, holds for $\nu_{2}$.

To verify that (1) holds for $\nu_{2}$ we require a finite collection of generating Markov partitions $\mathscr{R}=\mathscr{R}_{0}, \mathscr{R}_{1}, \ldots, \mathscr{R}_{k}$ with the property that the intersection of the boundaries of all the partitions is empty: $\bigcap\left(\partial \mathscr{R}_{i}\right)=\varnothing$. Such a collection can be constructed using a slight modification of the construction of Markov partitions given by Sinai [S2] in which a cover of $M$ by rectangles is modified an arbitrarily small amount to obtain a cover by rectangles whose boundaries map in the way required of a Markov partition, and then these rectangles are divided to produce a partition. Select a collection of covers of $M$ by small rectangles the boundaries of which are smooth, and so that the intersection of the boundaries of the different covers is empty. (That a finite number of covers suffices is seen by an argument about the dimension of the intersections of the boundaries. In fact, since the final step of Sinai's construction is a "cutting up", which adds extra boundary, it is necessary to require that slightly "extended" boundaries of the covers have intersections disjoint.) Perform Sinai's construction on the various covers moving them so little that their boundaries still do not intersect.

Now $\nu_{2}$ can be written as a convex combination of measures $\nu_{\alpha}$ each supported on the (disjoint) sets obtained as intersections of the form $\partial \mathscr{R}_{0} \cap \partial \mathscr{R}_{1}^{e_{1}} \cap$ $\partial \mathscr{R}_{2}^{e_{2}} \cap \cdots \cap \partial \mathscr{R}_{k}^{e_{k}}$, where the exponents $e_{i}$ take values +1 and -1 and indicate whether the set or its complement is to be used. Since the intersection with all the $e_{i}=+1$ is empty, each $\nu_{\alpha}$ gives zero mass to one of the sets $\partial \mathscr{R}_{i}$. Using this partition $\mathscr{R}_{i}$ and the argument of Lemma 4.2 to show that (1) holds for $\nu_{\alpha}$ completes the proof.

\subsection{Theorem. The system $(M, f, \mu)$ has a rate function given by}

$$
K(\nu)= \begin{cases}-h_{\nu}(f)-\int \phi^{u} d \nu & \text { if } \nu \in \mathscr{M}_{f}(M), \\ +\infty & \text { otherwise. }\end{cases}
$$

Proof. Since $\mu\left[L_{n} \in A\right]=\int \mu_{z}^{\xi}\left[L_{n} \in A\right] d \mu(z)$, and for $A$ closed (open) Lemma 4.2 gives upper (lower) bounds for $\mu_{z}^{\xi}\left[L_{n} \in A\right]$ which hold uniformly for $z$ outside a $\mu$-null set, the existence of the rate function follows. Also, the formula for $K(\nu)$ is a consequence of the two preceding lemmas.

4.5. Lemma. $m_{x}^{*}$ and $\mu(\cdot \mid \xi)(x)$ are equivalent, uniformly for $x$ in a set of full $\mu$-measure.

Proof. A proof of this lemma appears in Ledrappier [L], where weaker hypotheses than ours are assumed. One need only verify that Ledrappier's proof provides a uniform equivalence in the case of Anosov diffeomorphism. 
Define a metric on $\mathscr{M}(M)$ by selecting a sequence $\left\{\phi_{j}\right\}$ in the unit ball of $C(M, \mathbf{R})$ and setting, for $\mu, \nu$ in $\mathscr{M}(M)$,

$$
\rho(\mu, \nu)=\sum_{j=0}^{\infty} 2^{-j}\left|\mu\left(\phi_{j}\right)-\nu\left(\phi_{j}\right)\right| .
$$

If $A \subset \mathscr{M}(M)$ is closed, write, for $\delta>0, A^{\delta}=\{\nu: \operatorname{dist}(\nu, A) \leq \delta\}$. If $A \subset \mathscr{M}(M)$ is open and $\delta>0$, set $A_{\delta}=\{\nu: \operatorname{dist}(\nu,(\mathscr{M}(M) \backslash A))>\delta\}$.

Recall that $C_{\xi}(x)$ denotes the set in $\xi$ containing $x$, and is uniquely defined for $x \notin \partial \mathscr{R}$. If $x, x^{\prime}$ lie in the same rectangle $R_{i}$ of $\mathscr{R}$ then the map $h_{x, x^{\prime}}$ takes $C_{\xi}(x)$ to $C_{\xi}\left(x^{\prime}\right)$ and is defined by $h_{x, x^{\prime}}(y)=\left[y, x^{\prime}\right]$.

4.6. Lemma. For each $\delta>0$ there is an $N$ so that if $x$ and $x^{\prime}$ belong to $\operatorname{int}\left(R_{i}\right)$ for some $i$, and $n>N$ then:

(i) $h_{x^{\prime} x}\left(\left[z: L_{n}\left(z \in A^{\delta}\right)\right] \cap C_{\xi}\left(x^{\prime}\right)\right) \supset\left[z: L_{n}(z) \in A\right] \cap C_{\xi}(x)$ and

(ii) $h_{x^{\prime} x}\left(\left[z: L_{n}(z) \in A_{\delta}\right] \cap C_{x}\left(x^{\prime}\right)\right) \subset\left[z: L_{n}(z) \in A\right] \cap C_{\xi}(x)$

where in (i) $A$ is closed and in (ii) $A$ is open.

Proof. Postponed to the end of this section.

Proof of Theorem 4.1. Since $\mu\left(R_{i}\right)>0$ for each $i$, the sets $R_{i} \cap V$ are all nonempty. (The set $V$ is defined in Proposition 4.2.) For each closed set $A \subset \mathscr{M}(M)$ and $x \in M$, select a point $x^{\prime} \in V$ which is in the interior of some rectangle $R_{i}$ containing $x$. Denoting $\left\{y: L_{n}(y) \in A\right\}$ by $\left[L_{n} \in A\right]$, we have for $\delta>0$ and $n$ sufficiently large,

$$
\begin{aligned}
m\left(\left[L_{n} \in A\right] \mid \xi\right)(x) & \leq K_{1} m_{x}^{*}\left[L_{n} \in A\right], \quad \text { by equivalence of } m \mid \xi \text { and } m^{*}, \\
& \leq K_{1} m_{x}^{*}\left(h_{x^{\prime} x}\left[L_{n} \in A^{\delta}\right]\right), \quad \text { by Lemma } 4.6(\mathrm{i}), \\
& \leq K_{1} K_{2} m_{x^{\prime}}^{*}\left[L_{n} \in A^{\delta}\right], \quad \text { by Anosov's Theorem, } \\
& \leq K_{1} K_{2} K_{3} \mu\left(\left[L_{n} \in A^{\delta}\right] \mid \xi\right)\left(x^{\prime}\right), \quad \text { by Lemma 4.5. }
\end{aligned}
$$

Here the constants $K_{i}$ are independent of $x$ and $x^{\prime}$. Because $x^{\prime} \in V$ and $K$ is a rate function for $\left(M, f, \mu(\cdot \mid \xi)\left(x^{\prime}\right)\right)$ we have, for each $\delta, \varepsilon>0$ and $n$ sufficiently large, that

$$
m\left(\left[L_{n} \in A\right] \mid \xi\right)(x) \leq \exp \left(-n\left(\inf \left\{K(\nu): \nu \in A^{\delta}\right\}+\varepsilon\right)\right) .
$$

Thus, for each $\delta, \varepsilon>0$,

$$
\begin{aligned}
\limsup n^{-1} \log m\left[L_{n} \in A\right] & =\limsup n^{-1} \log \int m_{x}^{\xi}\left[L_{n} \in A\right] d m(x) \\
& \leq-\inf \left\{K(\nu): \nu \in A^{\delta}\right\}+\varepsilon
\end{aligned}
$$

Since $\inf _{\delta>0}\left\{-\inf \left\{K(\nu): \nu \in A^{\delta}\right\}\right\} \leq-\inf \{K(\nu): \nu \in A\}$, by the lower semicontinuity of $K$, this last inequality shows that $K$ is a lower deviation function for the system $(M, f, m)$. 

large,

If $B \subset \mathscr{M}(M)$ is open, we argue as above: If $\delta>0$ and $n$ is sufficiently

$$
\begin{aligned}
m\left(\left[L_{n} \in B\right] \mid \xi\right)(x) & \geq K_{1}^{-1} m_{x}^{*}\left[L_{n} \in B\right] \\
& \geq K_{1}^{-1} m_{x}^{*}\left(h_{x^{\prime} x}\left[L_{n} \in B_{\delta}\right]\right) \\
& \geq K_{1}^{-1} K_{2}^{-1} m_{x^{\prime}}^{*}\left[L_{n} \in B_{\delta}\right] \\
& \geq\left(K_{1} K_{2} K_{3}\right)^{-1} \mu\left(\left[L_{n} \in B_{\delta}\right] \mid \xi\right)\left(x^{\prime}\right) .
\end{aligned}
$$

Take $x^{\prime} \in V$ and integrate $m(\cdot \mid \xi)$ as above to conclude that for each $\delta>0$, $\liminf n^{-1} \log m\left[L_{n} \in B\right] \geq-\inf \left\{K(\nu): \nu \in B_{\delta}\right\}$. Since $\inf _{\delta>0} \inf _{B_{\delta}}(K(\nu)) \leq$ $\inf _{B}(K(\nu))$, this last inequality shows that $K$ is an upper deviation function for $(M, f, m)$ as well, completing the proof of Theorem 4.1.

Proof of Lemma 4.6. The proof depends on the following estimate (see [B]): There exist constants $C>0$ and $\gamma \in(0,1)$ so that if $y \in W_{\varepsilon}^{s}(x)$ and $j>0$, then

$$
d\left(f^{j}(y), f^{j}(x)\right)<\gamma^{j} C d(y, x) .
$$

The proof of the assertions of the lemma reduce to proving that $\rho\left(L_{n}(x), L_{n}(y)\right)$ goes to zero uniformly for $x \in M$ and $y \in W_{\varepsilon}^{s}(x)$. Using the triangle inequality one obtains the estimate:

$$
\begin{aligned}
\rho\left(L_{n}(x), L_{n}(y)\right) \leq & n^{-1} \sum_{k=0}^{T} \sum_{j=0}^{M} 2^{-j}\left|\phi_{j}\left(f^{k}(x)\right)-\phi_{j}\left(f^{k}(y)\right)\right| \\
& +n^{-1} \sum_{k=T+1}^{n-1} \sum_{j=0}^{M} 2^{-j}\left|\phi_{j}\left(f^{k}(x)\right)-\phi_{j}\left(f^{k}(y)\right)\right| \\
& +n^{-1} \sum_{k=0}^{n-1} \sum_{j=M+1}^{\infty} 2^{-j}\left|\phi_{j}\left(f^{k}(x)\right)-\phi_{j}\left(f^{k}(y)\right)\right| .
\end{aligned}
$$

First select $M$ so the last term is small. Then select $\delta$ so that $\left|f_{j}(t)-\phi_{j}(s)\right|$ is small when $d(s, t)<\delta$ for $j=0,1,2, \ldots, M$. Finally pick $T$ so that $d\left(f^{j}(x), f^{j}(y)\right)<\delta$ when $x \in M, y \in W_{\varepsilon}^{s}(x)$ and $j>T$. This makes the second term small. Then pick $n$ so the first term is small.

4.7. Corollary. For each continuous $\phi: M \mapsto \mathbf{R}$ there is a function $K^{\phi}: \mathbf{R} \mapsto$ $[0, \infty]$ which is a rate function for the convergence of the trajectory averages $n^{-1} \sum_{0}^{n-1} \phi\left(f^{j}(x)\right)$. In particular, for each $\phi$ and $\varepsilon>0$,

$$
m\left\{x:\left|n^{-1} \sum_{0}^{n-1} \phi\left(f^{j}(x)\right)-\int \phi d \mu\right|>\varepsilon\right\} \text { decays exponentially as } n \mapsto \infty \text {. }
$$

Proof. Theorem 4.1 and the "homomorphism theorem," Proposition 2.1, followed by an appropriate selection of the set $A$ in the definition of deviation function. 
Remark. Theorem 4.1 provides more evidence in favor of a conjecture by Takahashi $[\mathrm{T}]$ which states that a system is structurally stable if it has a rate function which is an affine map.

\section{REFERENCES}

[A] D. V. Anosov, Geodesic flows on closed Riemannian manifolds with negative curvature, Proc. Steklov Inst. Math., vol. 90, Amer. Math. Soc., Providence, R. I., 1969.

[B] R. Bowen, Equilibrium states and the ergodic theory of Anosov diffeomorphisms, Lecture Notes in Math., vol. 470, Springer-Verlag, New York, 1975.

[DV] M. Donsker and S. S. R. Varadhan, Asymptotic evaluation of certain Markov process expectations for large time, part 4, Comm. Pure Appl. Math. (1983), 183-212.

[L] F. Ledrappier, Propriétés ergodiques des mesures de Sinai, Inst. Hautes Études Sci. Publ. Math. (to appear).

[ME] N. F. G. Martin and J. W. England, Mathematical theory of entropy, Addison-Wesley, Reading, Mass., 1981.

[O] S. Orey, Large deviations in ergodic theory, Seminar on Stochastic Processes, Evanston, 1984 (E. Cinlar et al., eds.), Birkhäuser, Boston, Mass., 1986, pp. 195-259.

[OP] S. Orey and S. Pelikan, Large deviation principles for stationary shifts, IMA preprint no. 227.

[P] J. B. Pesin, Characteristic Lyapunov exponents and smooth ergodic theory, Russian Math. Surveys 32 (1977), 55-114.

[Ra] M. Ratner, Markov partitions for Anosov flows on $n$-dimensional manifolds, Israel J. Math. 15 (1973), 92-114.

[R] D. Ruelle, Thermodynamic formulations, Addison-Wesley, London, 1978.

[S1] Ya. G. Sinai, Markov partitions and C-diffeomorphisms, Functional Anal. Appl. 2 (1968), 70-80.

[S2] _ Construction of Markov partitions, Functional Anal. Appl. 2 (1968), 245-253.

[T] Y. Takahashi, Entropy functional ( free energy) for dynamical systems and their random perturbations. Proc. Taniguchi Sympos. on Stochastic Analysis (Katata and Kyoto, eds.), Kinokuniga/ North-Holland, 1980.

[V] S. S. R. Varadhan, Large deviations and applications, SIAM CBMS-NSF Regional Conference in Applied Math, no. 46, SIAM, Philadelphia, Pa., 1984.

School of Mathematics, University of Minnesota, Minneapolis, Minnesota 55455

Department of Mathematical Sciences, University of Cincinnati, Cincinnati, Ohio 45221 